\title{
Self-Interacting Dark Matter
}

\author{
Benjamin D. Wandelt ${ }^{1}$, Romeel Davé ${ }^{2}$, Glennys R. Farrar ${ }^{3}$, Patrick C. \\ McGuire $^{4,5}$ David N. Spergel $^{2}$, and Paul J. Steinhardt ${ }^{1}$ \\ 1 Department of Physics, Princeton University, Princeton NJ 08544, USA \\ 2 Department of Astrophysics, Princeton University, Princeton NJ 08544, USA \\ 3 Department of Physics, New York University, New York, NY 10003 USA \\ 4 Zentrum für Interdisziplinäre Forschung, Universität Bielefeld, Bielefeld, Germany \\ 33615 \\ 5 Steward Observatory and Department of Physics, University of Arizona, Tucson, \\ AZ 85721
}

\begin{abstract}
Spergel and Steinhardt have recently proposed the concept of dark matter with strong self-interactions as a means to address numerous discrepancies between observations of dark matter halos on subgalactic scales and the predictions of the standard collisionless dark matter picture. We review the motivations for this scenario and discuss some recent, successful numerical tests. We also discuss the possibility that the dark matter interacts strongly with ordinary baryonic matter, as well as with itself. We present a new analysis of the experimental constraints and re-evaluate the allowed range of cross-section and mass.
\end{abstract}

\section{$1 \quad$ Why Interacting Dark Matter Particles?}

Cosmological models with a mixture of roughly $35 \%$ collisionless cold dark matter such as axions, WIMPs, or any other candidate interacting through the weak and gravitational forces only, and $65 \%$ vacuum energy or quintessence match observations of the cosmic microwave background and large scale structure on extra-galactic scales with remarkable accuracy [2,3]. However, a number of discrepancies have arisen between numerical simulations and observations on subgalactic scales. [4] We will address these now in turn.

Halo density profiles. Simulations of collisionless cold dark matter halos (CCDM) [1, 6, 6] consistently show cuspy halo density profiles similar to the generalized Navarro-Frenk-White [0] (hereafter NFW) profile

$$
\rho(r)=\rho_{0} \frac{r_{s}^{3}}{r^{\alpha}\left(r+r_{s}\right)^{3-\alpha}}
$$

where $r_{s}$ is a scale radius, which is related to the virial radius $r_{h}$ of the halo via the concentration parameter $c=r_{h} / r_{s}$. This profile has the feature that it diverges as $\rho \sim r^{-\alpha}$ as $r \rightarrow 0$.

The exact value for $\alpha$ found in simulations is a matter of some debate NFW originally found $\alpha \simeq 1$, while more recent, higher resolution simulations find somewhat steeper divergences, $\alpha \simeq 1.5$. This is unlikely to be an artifact; if anything, resolution and discreteness effects lead to simulations underestimating 
the steepness of the central density profile. Biases can occur when fitting (1) to simulated halos. For example, the fact that there is a finite number of particles per halo make the choice of the halo center somewhat arbitrary. Any departures from the "true" center will give the illusion that the density profile is less cuspy than it actually is.

Observationally, gravitational lensing in clusters and the presence of disks in galactic halos make it possible to infer their mass distributions. Observations of low surface brightness galaxies (LSB) and dwarfs, chosen for their small mass-tolight ratios, indicate cores of galactic halos with shallow density profiles [8, [9,10]. Similarly, there is some evidence that the central regions of cluster halos not associated with luminous galaxies appear have flat density profiles $\left(\rho=r^{0}\right.$ as $r \rightarrow 0)$ [11].

More recently, preliminary results from high quality measurements of rotation curves in edge-on LSBs with thin disks [12] indicate a best fit of $\alpha \simeq 0.5$, more mildly divergent than and inconsistent with CCDM profiles.

Note that only exquisitely well-measured rotation curves simultaneously constrain both the concentration parameter $c$ and the slope $\alpha$ [13. In practice, observations do not resolve these parameters independently. This means that the problem of halo shapes can be rephrased in terms of $c$; observed halos tend to have smaller concentration $(c \simeq 6-8)$ than simulated ones $(c \simeq 20)$. The crucial point is that whether one focuses on slope or concentration, simulations and observations do not agree.

Excess of small scale structure. One of the most striking features of halos in high resolution CCDM simulations is that they are heavily populated with small subhalos or subclumps. The scarcity of observed satellite galaxies 115] could be interpreted as another failure of the CCDM paradigm on small scales.

Even if there are mechanisms which inhibit star formation in small clumps and thereby leave them dark, a large population of clumps may heat the galactic disk and even endanger its stability [15, 16, 17].

This is a delicate issue; there clearly is halo substructure in galaxies (satellites, dwarfs, high velocity clouds). Any proposed solution must reduce the number of satellites but guard against wholesale smoothing of the density field on small scales.

Tully-Fisher relation. Further evidence for less centrally concentrated halos comes from the failure of CCDM models to account for the zero-point of the Tully-Fisher relation. Mo and Mao 18 explain the surprisingly tight relation between the luminosity of disk galaxies and their maximum rotation speed in terms of the gravitational interactions between galaxy disk and halo. Their model requires the presence of a dark halo with concentration $c \simeq 3$, much less than $c \simeq 20$ which is found in simulations. Significantly, this pertains to all disk galaxies - not just LSBs and dwarfs.

Bar stability. In high surface brightness spiral galaxies, the persistence of bars implies low-density cores 19].

It is conceivable that these discrepancies are due to problems with current simulations, the quality of present observations or the omission of important 
astrophysical processes in the models. However, if any one of them persists, they may be an indication that the dark matter is not collisionless.

In this paper we argue that all of the discrepancies listed above may be resolved if the dark matter has strong self-interactions. If this proves to be the case, the study of fine-scale structure on subgalactic (kiloparsec) scales, a subject that has not been given much attention by particle physicists in the past, could prove to be a remarkably powerful and precise probe of the properties of dark matter.

\section{The Physics of Self-Interacting Dark Matter Halos}

Consider a CCDM halo. The particles in its center have small rms speeds (are "cold"). As subclumps sink into the larger halo, their outer, hotter, less tightly bound parts are tidally stripped and remain in the outer parts of the halo. If the cold centers of the clumps survive, they join the cold cusp of the halo. Because of the lack of two-body interactions (the gravitational potential is smooth) there is no heat transport into the cold CCDM cusp.

Virialized CCDM halos are not in thermal equilibrium. When interactions are present, however, the second law of thermodynamics requires that heat be transferred into the cold center from the outer halo to approach equilibrium. Initially, the increased rms speeds spread out the halo cusp into a smoother core. At the same time as the inner part is heated, the outer part is cooled, the cooler material moves in, is scattered and mixed, so that eventually the halo becomes isothermal at late times. Particles will be ejected due to two-body interactions, the halo loses heat and gravothermal collapse sets in, moderated by ongoing accretion of small subclumps, which continue to heat the halo.

An important fact to keep in mind is that the heat conduction coefficient does not have a simple dependence on the cross section. A traditional analysis $\grave{a}$ la Chapman and Enskog 20] expands about thermal equilibrium which is brought about by frequent interactions. In this regime, where the mean free path of the particles is much shorter than any characteristic length scale of the system, the heat conduction decreases with increasing cross-section, $\kappa(r) \propto \frac{\pi k_{B}}{\sigma} \sqrt{\frac{\left\langle v^{2}(r)\right\rangle}{\pi}}$.

As we will see below, the interactions discussed here are constructed to be in a different regime altogether. Here, the mean free path is of the order of the scale of the halo. In this regime, namely the small cross-section limit, heat transport increases as the cross-section increases. For this reason a numerical implementation of self-interacting dark matter is non-trivial and cannot be achieved by solving the standard equations of cosmological hydrodynamics. Also, because of the non-monotonic dependence on cross-section, a simple interpolation between the case of collisionless dark matter and the hydrodynamic limit is not sufficient.

The presence of scatterings has another important effect: the particle velocities become randomized. The random component of particle velocities in a collisionless fluid can be highly correlated, leading to non-spherical velocity ellipsoids [21]. Collisions erase these correlations, randomizing the motions, which 
leads to more spherical velocity ellipsoids. The resulting lack of a preferred direction should isotropize the inner parts of halos where scattering is important.

Spergel and Steinhardt [1] postulated a self-interaction between dark matter particles with strength which encompassed the range

$$
s=\frac{\sigma_{D D}}{M}=8 \times 10^{-25}-1 \times 10^{-23} \mathrm{~cm}^{2} \mathrm{GeV}^{-1}=0.5-6 \mathrm{~cm}^{2} \mathrm{~g}^{-1}
$$

many orders of magnitude stronger than for CCDM particles. (The range quoted here is narrower than originally proposed in [1] because of additional constraints discussed below). A convenient dimensionless quantity measuring the strength of the interaction at the solar circle is the optical depth $\tau=s / s_{\text {solar }}$ where $s_{\text {solar }}=28.4 \mathrm{~cm}^{2} / \mathrm{g}$. Unit optical depth corresponds to a mean free path of order the diameter of the solar orbit $\lambda_{8 \mathrm{kpc}}=16 \mathrm{kpc}$ at the densities of the solar neighborhood $\left(\rho_{8 \mathrm{kpc}} \simeq 10^{5} \rho_{\text {crit }} \simeq 7 \times 10^{-25} \mathrm{~g} / \mathrm{cm}^{3}\right)$. One can then estimate the size of the optically thick region $R_{o p t} \simeq 8 \tau \mathrm{kpc}$.

The range of $s$ originally quoted in [1] was chosen to cover a span of optical depths straddling unity. On the optically thin side, subhalos are destroyed by spallation in which particles are scattered out of the subhalo one-by-one almost every time there is an impact with a hotter particle from the surrounding halo. On the optically thick side, subhalos are destroyed by evaporation in which the subhalo absorbs all the kinetic energy from nearly every incoming hot particle of the surrounding halo, heats up and expands. Either mechanism suffices for the purpose of destroying dwarf halos in galaxies. However, a problem with evaporation is that it is so efficient that galaxy halos in massive clusters probably cannot survive, which conflicts with observations. Eq. (2) takes account of this problem by shaving off the optically thick regime from the range of $s$ found in [1].

\section{$3 \quad$ Numerical Studies}

To summarize, the qualitative effects of introducing self-interacting dark matter (SIDM) in the regime of cross-sections in (2) are:

- Heat transport leading to modified halo profiles and core collapse on time scales larger than the Hubble time.

- Isotropization of the velocity tensor in dense regions and hence more spherical shapes of the inner parts of halos.

- Limited destruction of halo substructure through spallation.

Intriguingly, the observations we listed in the introduction suggest precisely this sort of behavior.

This has motivated several authors [22,23,24,25,26,27, to study the effects of self-interactions numerically. Apart from simulations which are done in the hydrodynamic limit 24,25, all of these find that SIDM halos contain smooth cores. However, the longevity of these cores and the time-scale for gravitational collapse are still a matter of debate. 


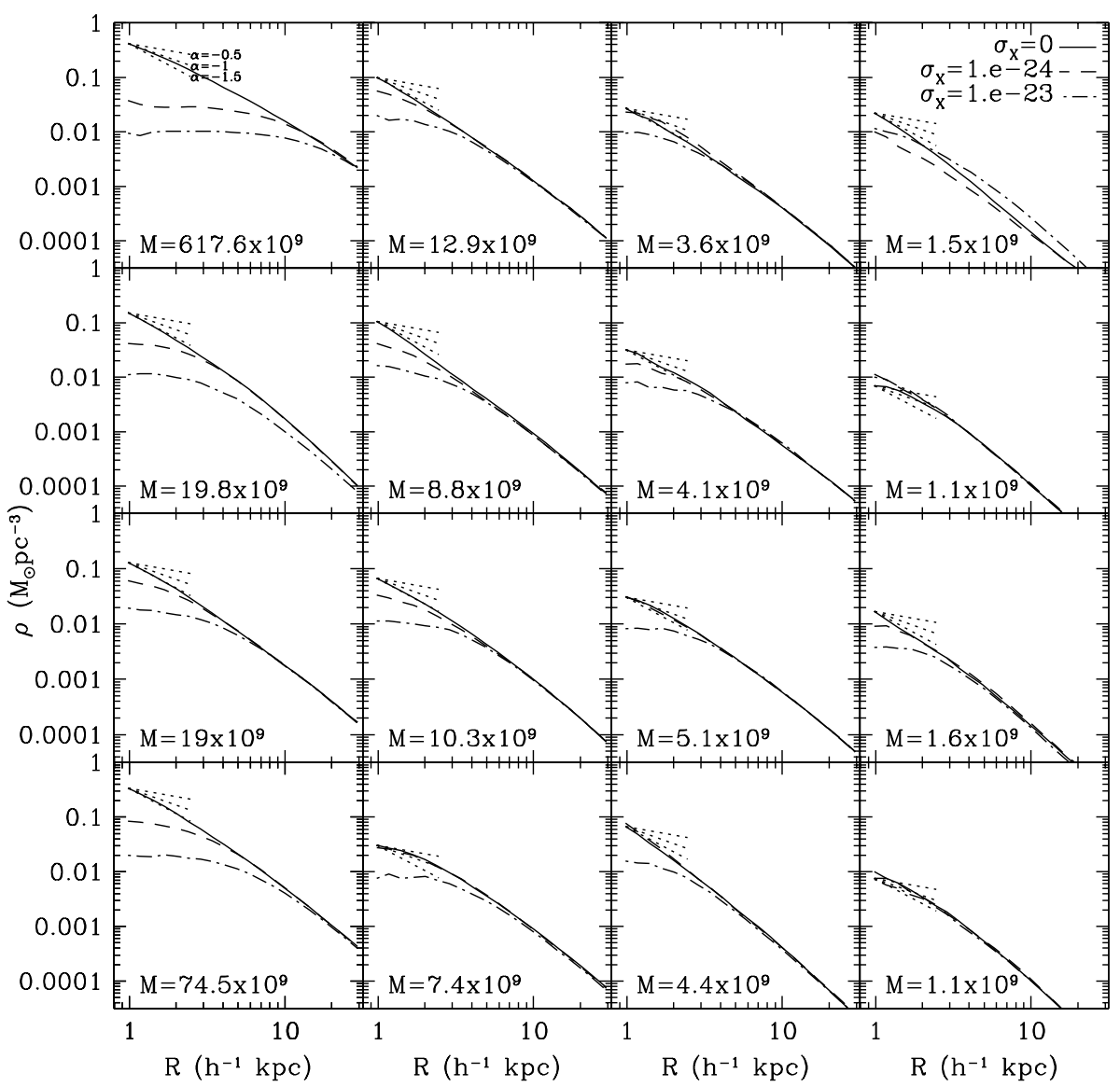

Fig. 1. Comparison of halo profiles for non-interacting (solid) and self-interacting dark matter with cross-sections $\sigma_{D D}=10^{-24} \mathrm{~cm}^{2} \mathrm{GeV}^{-1}$ (dashed) and $\sigma_{D D}=$ $10^{-23} \mathrm{~cm}^{2} \mathrm{GeV}^{-1}$ (dot-dashed). Halos are chosen from a descending range of masses, with the four largest halos in our simulation shown in the leftmost column Three short rays corresponding to logarithmic slopes $0.5,1$ and 1.5 are shown to guide the eye in the inner part of the halo

In particular, Kochanek and White simulate isolated halos and find fast evolution towards gravothermal collapse for higher mass halos [26]. Caveats of their simulations include that 1) they begin with steep, high-density profiles which are already cuspy before the interactions are turned on, which leads to fast evolution because the central density starts from artificially high values, 2) they do not include accretion, which ought to moderate gravothermal collapse and 3) the cross-section they simulate are high. For a Milky Way type galaxy $\left(R_{0} \simeq 10 R_{\text {disk }} \simeq 35 \mathrm{kpc}, M_{0} \simeq 7 \times 10^{11} M_{\odot}\right)$ their simulations cover a range from $s \simeq 2.5-83 \mathrm{~g} / \mathrm{cm}^{2}$. They observe core collapse for cross-sections above our 


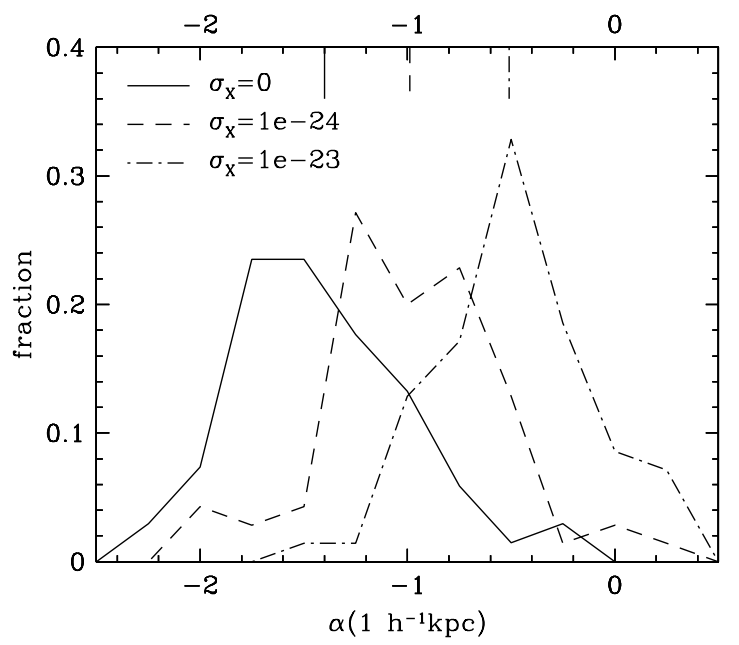

Fig. 2. Histogrammed distribution of inner halo slopes $\alpha$ for non-interacting (solid) and self-interacting dark matter with cross-section $\sigma_{D D}=10^{-24} \mathrm{~cm}^{2} \mathrm{GeV}^{-1}$ (dashed) and $\sigma_{D D}=10^{-23} \mathrm{~cm}^{2} \mathrm{GeV}^{-1}$ (dot-dashed). We only include halos with more than 1000 particles, corresponding to masses greater than $3.6 \times 10^{9} M_{\odot}$

range (2). Burkert 23] has performed simulations comparable to Kochanek and White's, and his results suggest significantly longer lived cores. Yoshida et al. [27) compute the halo profile of a cluster from cosmological initial conditions. They, too, find large cores which persist until today.

For our simulations, we modified the freely available GADGET tree N-body code [28] to include scatterings between dark matter particles using Monte Carlo techniques similar to [26]. The simulations begin with cosmological initial conditions in the Lambda model currently favored by the majority of observations $\left(\Omega_{0}=1=\Omega_{m}+\Omega_{\Lambda}, \Omega_{\Lambda}=0.7\right)$. We follow the evolution of dark matter, represented by $128^{3}$ particles in a box with $4 h^{-1} \mathrm{Mpc}$ on the side and $1 h^{-1} \mathrm{kpc}$ spatial resolution. Periodic boundary conditions ensure that we model the continuing accretion of halos correctly.

We simulate interaction strengths of $s \simeq 0.06,0.6,6$ and $60 \mathrm{~cm}^{2} / \mathrm{g}$. Out of these the smallest value of $s$ produced results that are barely distinguishable from collisionless dark matter. The largest cross-section considered significantly suppresses the formation of halos, and those that do form are much too large and diffuse. But for the two intermediate cases our galactic halos consistently developed constant density cores.

Figure 1 compares the profiles of halos from three of our simulations which assume different cross-sections but which are otherwise identical. It is clear that for intermediate cross-sections, within the range suggested by Spergel and Steinhardt, core collapse has not set in. Consistent with our previous discussion of 
the optically thin regime, we find evidence that the stronger the self-interactions are, the smoother the halos become.

Figure 2 shows the histograms of inner slopes measured for all halos with more than 1000 particles in each of the 3 simulations. It is clear that in the regime we are exploring, increasing the cross-section smoothes the halo profiles.

These and other results from our simulations will be discussed in more detail in a forthcoming publication 29].

\section{Could Dark Matter Consist of an Exotic, Neutral, Stable Hadron?}

If self-interactions are responsible for resolving the issues of the halo profile and galactic substructure, then the ratio of the dark matter-dark matter crosssection $\sigma_{D D}$ to the mass of the dark matter particle must lie in the range given in Eq. (2). Curiously, the ratio for ordinary hadrons, such as neutrons and protons, lies in roughly this same range, $\mathcal{O}\left(10^{-23} \mathrm{~cm}^{2} / \mathrm{GeV}\right)$ at low energies. One natural possibility is that the dark matter belongs to the hidden sector of a supergravity or superstring model with hidden gauge interactions analogous to ordinary gluon exchange. A second possibility is that the dark matter is composed of exotic hadrons - neutral, stable particles which interact through the strong force with ordinary quarks and gluons as well as with themselves. In this case, the ratio of scattering cross-section to mass for self-scattering $(s)$ might be comparable to the ratio for dark matter-nucleon scattering. We should emphasize that the selfinteraction proposal does not require that there be strong interactions between dark matter and ordinary hadrons; it suffices if the dark matter interacts strongly with itself. However, the fact that the cross-section required to solve the halo profile problem is so similar to the cross-section for the familiar strong interaction makes it impossible to resist considering the possibility.

If the exotic hadron is neutral and stable and does not radiate photons or other massless mesons, then it will act like dissipationless, collisional dark matter. Possible examples include the quark-gluino bound state, the $S 0$ (in some models, the lightest supersymmetric stable particle with odd $R$-parity and unit baryon number) 30,31] or strangelets [32]. Alternatives are non-hadronic exotics which might also be arranged to have the desired value of $\sigma_{D D} / m$, such as gauge singlet mesons [33], and $Q$-balls [34]. Of these, the first three naturally have values of $s=\sigma_{D D} / m$ which lie in the desired range, Eq. (2). Another condition is that the exotic hadron must not bind to light nuclei. Otherwise, the particle would efficiently convert hydrogen and helium to exotic nuclei during nucleosynthesis and would be found plentifully. Binding to light nuclei may be sufficiently suppressed if the particle has quantum numbers that forbid simple pion exchange with ordinary nuclei, as might occur if the particle is an isospin singlet. Yet a further constraint is that the cosmic abundance must account for the observed matter density. If the particle has similar mass and interactions to ordinary hadrons, perhaps a natural explanation can be found for why the cosmic energy density in exotic hadrons is comparable that of ordinary hadrons. 
One may suppose that the notion of exotic hadron is easily discounted. Surely the particle is already ruled out by existing cosmological and astrophysical constraints or by existing accelerator experiments or dark matter detectors or searches for exotic nuclei. Surprisingly, the story is not nearly so simple. For example, if the particle is strongly interacting and similar to a neutron in most characteristics, it evades all currently published constraints known to the authors. During nucleosynthesis, its kinetic energy density is too small to affect the cosmic expansion rate and, if it does not bind to light nuclei, it has no effect on ordinary nuclear abundances or evades terrestrial searches for unusual nuclei. Only an undetectably small fraction of the particles are captured by stars, such as the Sun, so they have no significant effect on stellar evolution, supernovae, etc. The scattering cross-section for dark matter on ordinary protons and neutrons $\sigma_{D p}$ would presumably be close to the dark matter-dark matter cross-section, $\sigma_{D D}$, so one concern is that halo dark matter collides with ordinary baryons in the disk, creating excess high energy cosmic rays and destabilizing the disk. We find that these considerations do not rule out the exotic hadron scenario, either. In cases where the mass and interaction strength is similar to the more abundantly produced neutrons, it is difficult to exclude them with accelerator experiments.

Hence, one is led to consider dark matter searches. However, most dark matter searches are aimed at weakly interacting particles (WIMPS), such as axions and supersymmetric neutralinos, which can penetrate the atmosphere and deep underground unimpeded. Consequently, the detectors are placed at ground level or in mines. Strongly interacting particles interact in the upper atmosphere and will thermalize with the air before they reach ground level. For a $1 \mathrm{GeV}$ particle, say, the incoming kinetic energy is $1 \mathrm{keV}$ (corresponding to a virial velocity of $300 \mathrm{~km} / \mathrm{s}$ ) and a local density of $0.4 \mathrm{GeV} / \mathrm{cm}^{3}$. If the particle is strongly interacting, then the average kinetic energy is an undetectable $30 \mathrm{meV}$ by the time the particles reach ground level.

¿From these examples, it is clear that the best way to search for strongly interacting particles is in balloons or in space with detectors with little or no shielding and low energy threshold. A decade ago, Starkman et al. [35] surveyed all current observations and experiments and found that the combination of cross-section and mass suggested by Eq. (2) is not constrained for masses near $1 \mathrm{GeV}$ or so, and no new constraints have been published since - until now.

Figure 3 represents our new analysis of the constraints on strongly interacting dark matter [31. This plot assumes a spin-independent cross-section for scattering of dark matter from nuclei with atomic mass $A$ that is $\sigma_{D A}=$ $A^{2}\left[m_{r e d}(A) / m_{r e d}(1)\right]^{2} \sigma_{D p}$, as considered by Starkman et al. [35]. It is straightforward to modify the plot for spin-dependent cross-sections or cases with different $A$-dependence, as might be expected for some of our candidates. The grey strip represents the range of $\sigma_{D D} / m$ given in Eq. (2), which one might imagine is roughly similar to the dark matter-proton cross-section $\sigma_{D p} / m$. The plot has three new features compared to earlier, similar exclusion plots: 


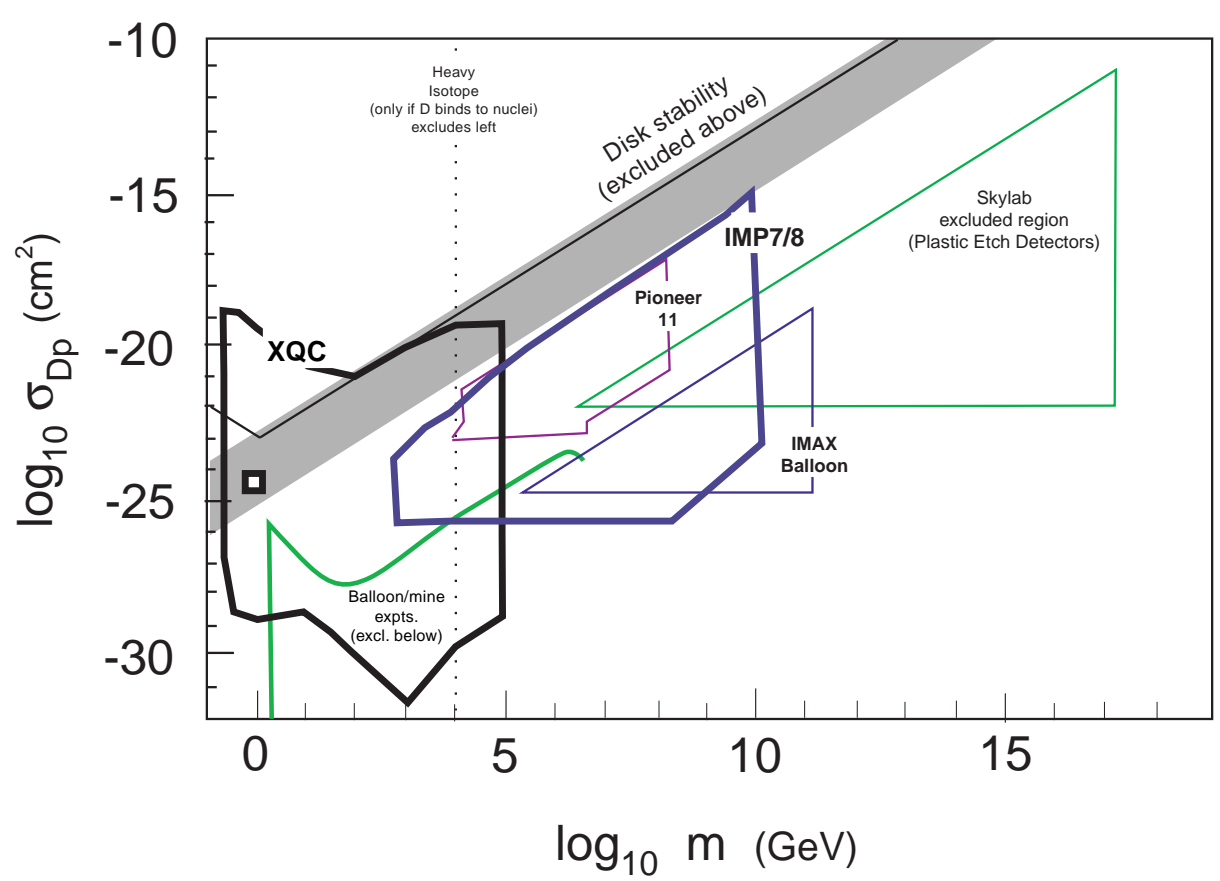

Fig. 3. Plot of the dark matter-proton scattering cross section $\sigma_{D p}$ versus dark matter particle mass $m$ showing current experimental limits. The constraints from the XQC, IMP7/8 and IMAX experiments are new; the constraints from Pioneer and Skylab experiments have been re-evaluated. The grey region shows the range of dark matter-dark matter cross-section $\sigma_{D D}$ in Eq. (2). The strip is displayed to study the possibility that dark matter consists of exotic hadrons in which $\sigma_{D D} / m$ and $\sigma_{D p} / m$ are comparable. The square marks the values corresponding to a nucleon, which is similar to what is expected for some of the most attractive candidates for dark matter. The new results appear to rule out the low mass range, but allow masses greater than $10^{5} \mathrm{GeV}$.

(1) Some important corrections [38] have been applied to constraints from older experiments (Pioneer 11 and Skylab) compared to Starkman et al. [35]. The corrections reduce the exclusion region at large cross-section; earlier analyses had failed to take proper account of shielding. We also argue that the neutron star bound in [35] does not apply in the limit of large cross-sections [31]. The re-evaluation opens up a range of the grey-striped region for masses between $10^{4}$ to $10^{17} \mathrm{GeV}$.

(2) We publish for the first time results of a complete analysis of the IMP7/8 cosmic ray silicon detector satellite 43, 36, 38 and the IMAX balloon-borne SIMP (strongly interaction massive particle) detector [37, 38, 39].

(3) We present a provisional exclusion region for the X-ray Quantum Calorimeter (XQC) experiment [40], an experiment whose first results are not yet published 41 and that was not designed with dark matter in mind. Remarkably, this "inadvertent" dark matter detector provides perhaps the most important constraint, 
ruling out a large, previously unconstrained region of parameter space spanning particles in the $0.3 \mathrm{GeV}$ to $10^{5} \mathrm{GeV}$ range that includes the $S 0$ and low-mass strangelets. (A more sophisticated analysis 42 is likely to expand the exclusion region somewhat.) The key properties of $\mathrm{XQC}$ are that it has impressively sensitive pixels with a low energy threshold $(25 \mathrm{eV}$ for a substantial portion of the flight, compared to $160 \mathrm{keV}$ for IMP7) a significant detecting area, minimal atmospheric overburden, and negligible material in front of its 1 sr field-of-view. The detector consists of 33 pixels of $1 \mathrm{~mm}^{2}$ area, each with an x-ray absorber (7000 Angstroms of $\mathrm{HgTe}$ ) deposited on 15 microns of silicon attached to silicon detector structure with an implanted thermometer. Both the HgTe and the silicon layers are active in detection; silicon dominates the low-mass region and HgTe is more important for excluding the high mass region. Although the detector flew on a sounding rocket at an altitude of $170 \mathrm{~km}$ for only a few minutes, the low event rate (probably due to x-ray sources) places strong constraints on dark matter candidates. Stronger limits can be obtained with subsequent flights.

\section{Conclusion}

We find that recent simulations 27,29 based on the self-interaction proposal of Spergel and Steinhardt with self-interactions of strength $0.1 \mathrm{~cm}^{2} / \mathrm{g}<s<$ $6 \mathrm{~cm}^{2} / \mathrm{g}$ lend strong support for the concept, producing results that fit observations significantly better than standard collisionless cold dark matter models. The favored dark matter candidates, axions and neutralinos, are effectively collisionless and, hence, are in some considerable jeopardy. The Spergel-Steinhardt proposal has stimulated the interesting possibility that dark matter consists of particles that interact through the strong force with ordinary matter. Our reevaluation of constraints leads us to conclude that the exotic hadron possibility is now ruled out for a substantial range of masses near $1 \mathrm{GeV}$ and cross-sections near $10^{-24} \mathrm{~cm}^{2}$, eliminating some of the most attractive possibilities. At the same time, the re-evaluation has re-opened a region encompassing larger masses and cross-sections previously thought to be ruled out.

\section{Acknowledgements}

We thank Lyman Page for bringing the XQC experiment to our attention and J. Dalcanton, N. Yoshida and D. McCammon for sharing their results with us prior to publication. We are also grateful to the participants of the Princeton Monday cosmology meetings for many useful criticisms. RD and DNS are supported by the NASA ATP grant NAG5-7066. DNS and BDW are supported by the NASA MAP/MIDEX program. This work was supported in part by Department of Energy grant DE-FG02-91ER40671 (PJS).

\section{References}

1. D.N. Spergel, P.J. Steinhardt: Phys. Rev. Lett. 84, 3760 (2000) 
2. N.A. Bahcall, J.P. Ostriker , S. Perlmutter, and P.J. Steinhardt: Science 284, 1481 (1999)

3. L. Wang, R.R. Caldwell, J.P. Ostriker, and P.J. Steinhardt: Ap. J. 530, 17 (2000)

4. S. Ghigna, B. Moore, F. Governato, G. Lake, T. Quinn, J. Stadel: Density Profiles and Substructure of Dark Matter Halos: "Converging" Results at Ultra-High Numerical Resolution, astro-ph/9910166

5. J.F. Navarro, C.S. Frenk, S.D.M. White: Ap. J. 462, 562 (1996)

6. A.A. Klypin, A.V. Kravtsov, O. Valenzuela, F. Prada: Ap. J. 522, 82 (1999)

7. A. Klypin: Lecture at the Summer School "Relativistic Cosmology: Theory and Observations", Como, Italy (2000)

8. R.A. Flores, J.A. Primack: Ap. J. 427, L1 (1994)

9. W.J.G. De Blok, S.S. Mc Gaugh: MNRAS 290, 533 (1997)

10. J. Dalcanton, R. Bernstein: MNRAS 290, 533 (1997)

11. J.A. Tyson, G.P. Kochanski, I.P. Dell'Antonio: Ap. J. 498, L107 (1998)

12. J. Dalcanton: Washington University, private communication (2000)

13. F.C. van den Bosch, B.E. Robertson, J.J. Dalcanton, W.J.G. de Blok: Constraints on the Structure of Dark Matter Halos from the Rotation Curves of Low Surface Brightness Galaxies, astro-ph/9911372

14. J. Miralda-Escude: A Test of the Collisional Dark Matter Hypothesis from Cluster Lensing, astro-ph/0002050

15. B. Moore, S. Ghigna, F. Governato, G. Lake, T. Quinn, J. Stadel, P. Tozzi: Ap. J. 524, L19 (1999)

16. G. Toth, J.P. Ostriker: Ap. J. 389, 5 (1992)

17. M. Weinberg: MNRAS 299, 499 (1998)

18. H.J. Mo, S. Mao: Tully-Fisher Relation and its Implications for Halo Density Profile and Self-interacting Dark Matter, astro-ph/0002451

19. V.P. Debattista and J. Sellwood: Ap. J. 493, L5 (1998)

20. R.L. Liboff: Kinetic Theory: Classical, Quantum, and Relativistic Descriptions (Prentice Hall, London 1990)

21. J. Binney, S. Tremaine: Galactic Dynamics (Princeton University Press, Princeton 1987)

22. S. Hannestad: Galactic Halos of Self-Interacting Dark Matter, astro-ph/9912558

23. A. Burkert: The Structure and Evolution of Weakly Self-Interacting Cold Dark Matter Halos, astro-ph/0002409

24. B. Moore, S. Gelato, A. Jenkins, F.R. Pearce, V. Quills: Ap. J. Lett. 535, 21 (2000)

25. N. Yoshida, V. Springel, S.D.M. White, G. Tormen: Collisional dark matter and the structure of dark halos, astro-ph/0002362

26. C.S. Kochanek,and M. White: A Quantitative Study of Interacting Dark Matter in Halos, astro-ph/0003483

27. N. Yoshida, V. Springel, S.D.M. White, G. Tormen: Weakly Self-Interacting Dark Matter and the Structure of Dark Halos, astro-ph/0006134

28. V. Springel, N. Yoshida, S.D.M. White: http://ibm-2.MPA-Garching.MPG.DE/gadget/

29. R. Davé, D.N. Spergel, P.J. Steinhardt, B.D. Wandelt: Halo Properties in Cosmological Simulations of Self-Interacting Cold Dark Matter, astro-ph/0006218

30. G.R. Farrar: Phys. Rev. Lett. 53, 1029 (1984); Nucl.Phys.Proc.Suppl. 62, 485 (1998)

31. P.J. Steinhardt, D.N. Spergel, G. Farrar, P.C. McGuire, B.D. Wandelt: in preparation

32. R.L. Jaffe, W. Busza, J. Sandweiss, F. Wilczek: hep-ph/9910333 
33. M.C. Bento, O. Bertolami, R. Rosenfeld, L. Teodoro: Self-Interacting Dark Matter and Invisibly Decaying Higgs, astro-ph/0003350

34. A. Kusenko and M. Shaposhnikov: Phys. Lett. B 418, 46 (1998)

35. G.D. Starkman, A. Gould, R. Esmailzadeh, S. Dimopoulos: Phys. Rev. D 41, 3594 (1990)

36. P.C. McGuire, T. Bowen, R. Mewaldt, P. Steinhardt: in preparation

37. P.C. McGuire and T. Bowen: Proc. 5th October Astrophysics Conf. in College Park, Maryland on Dark Matter, AIP, ed. by S.S. Holt and C.L. Bennett, Vol. 336, p. 53 (1994)

38. P.C. McGuire: Balloon-Borne Direct Search for Ionizing Massive Particles as a Component of the Dark Galactic Halo Matter, Ph.D. dissertation, University of Arizona (1994)

39. P.C. McGuire, T. Bowen: in preparation

40. D. McCammon et al.: NIM A 370 , 266 (1996)

41. D. McCammon: University of Wisconsin, private communication (2000)

42. D. McCammon et al.: in preparation

43. R. Mewaldt: California Institute of Technology, private communication (2000) 